山्山FRANÇAISE

$>\mathrm{DE}$

$\stackrel{1}{\simeq}$ PÉDAGOGIE
Revue française de pédagogie

Recherches en éducation

199 | avril-mai-juin 2017

L'histoire des disciplines : un champ de recherche en mutation

\title{
Les professeurs d'ENNA et leur rôle dans la structuration de la discipline « français » entre 1945 et 1960
}

ENNA Teachers and their role in the structuring of "French" as a school subject between 1945 and 1960

Maryse Lopez

\section{OpenEdition}

Journals

\section{Édition électronique}

URL : https://journals.openedition.org/rfp/6085

DOI : $10.4000 / r f p .6085$

ISSN : 2105-2913

\section{Éditeur}

ENS Éditions

\section{Édition imprimée}

Date de publication : 30 juin 2017

Pagination : 81-94

ISBN : 979-10-362-0108-0

ISSN : 0556-7807

Référence électronique

Maryse Lopez, "Les professeurs d'ENNA et leur rôle dans la structuration de la discipline " français » entre 1945 et 1960 », Revue française de pédagogie [En ligne], 199 | avril-mai-juin 2017, mis en ligne le 30 juin 2020, consulté le 07 janvier 2022. URL : http://journals.openedition.org/rfp/6085 ; DOI : https:// doi.org/10.4000/rfp.6085 


\title{
Les professeurs d'ENNA et leur rôle dans la structuration de la discipline « français » entre 1945 et 1960
}

\author{
Maryse Lopez
}

\begin{abstract}
Mises en place en 1945, en même temps que les centres d'apprentissage chargés de préparer au CAP des jeunes majoritairement issus des classes de fin d'études primaires, les écoles normales nationales d'apprentissage (ENNA) ont pour mission de former leurs futurs enseignants. Parmi les professeurs ayant en charge l'enseignement du français, quelques personnalités proches de la direction de l'Enseignement technique et partageant son projet éducatif et culturel émergent. L'approche qui croise histoire et didactique permet de saisir le rôle joué par ces enseignants dans la genèse de la discipline «français » entre 1945 et 1959, au moment où elle se donne pour finalité la formation des futurs travailleurs.
\end{abstract}

Mots-clés (TESE) : formation initiale des enseignants, formation et enseignement professionnels, formateur, programmes d'étude, matière d'enseignement

\begin{abstract}
À la Libération, les centres de formation professionnelle créés sous Vichy se transforment en centres d'apprentissage ${ }^{1}$ rattachés à l'enseignement technique en 1949 (Troger, 1991)². Ancêtres des actuels lycées professionnels, ces établissements viennent s'ajouter aux autres écoles de l'enseignement technique déjà existantes que sont les collèges techniques et les écoles nationales professionnelles (ENP) ${ }^{3}$. Ces centres ont
\end{abstract}

1 Arrêté du 18 septembre 1944, JO n 88 du 4 octobre 1944, p. 864-876.

2 Loi $49-230$ du 21 février 1949, JO n 46 du 22 février 1949, p. 321-322.

3 Les ENP et les collèges techniques, EPCI (écoles pratiques du commerce et de l'industrie) jusqu'en 1941, deviendront lycées tech- pour mission de préparer en trois ans au certificat d'aptitude professionnelle (CAP) des élèves le plus souvent d'origine populaire et issus majoritairement des classes de fin d'études primaires et n'ayant pas pu accéder au collège technique. L'apparition de ces établissements dans le paysage scolaire ouvre la voie à une formation professionnelle scolarisée à grande échelle et conduit à distinguer l'enseignement technique court (les

niques en 1959. Ils constitueront l'enseignement technique long alors que les centres d'apprentissage, devenus collèges d'enseignement technique (CET) à la même date, constitueront l'enseignement technique court. Ils deviendront lycées d'enseignement professionnel (LEP) en 1976. Voir Pelpel \& Troger, 1993. 
centres d'apprentissage) de l'enseignement technique long (les collèges techniques et les ENP). Elle s'accompagne de la création de cinq écoles normales nationales d'apprentissage (ENNA), trois masculines et deux féminines, à Paris, Lyon, Nantes et Toulouse ${ }^{4}$. Ces écoles sont chargées de former, dans un même lieu, les futurs enseignants des disciplines générales et techniques des centres d'apprentissage. Le projet éducatif de ces écoles naissantes - former l'homme, le citoyen et le travailleur pour reprendre une formule en vogue à l'époque - est à resituer à la fois dans la tradition d'une vision humaniste de l'apprentissage (Brucy, 2005) et dans le contexte du plan Langevin-Wallon (1947) qui prône une articulation étroite entre apprentissage du métier et formation générale "afin d'avoir une action plus profonde sur l'enfant et d'éviter au métier de devenir une routine sans intérêt» (Sorel, 1997, p.212; Allart-Gondouin, 2016).

Les centres d'apprentissage et les ENNA s'avèrent être des lieux privilégiés pour promouvoir une culture technique moderne (Naville, 1948) et, plus généralement, porter un projet de rénovation pédagogique dans le contexte de l'après Seconde Guerre mondiale (Gal, 1946). Si le projet éducatif et culturel des ENNA a déjà été étudié (Tanguy, 1991; Troger, 1998; Terral, 2002; Lopez, 2014), l'étude des professeurs d'ENNA, recrutés par concours jusqu'au milieu des années 1980, et de leur rôle dans la genèse et la consolidation des disciplines de l'enseignement professionnel reste à faire. Dans cette perspective, nous nous intéresserons ici plus spécifiquement aux professeurs chargés d'enseigner la discipline "français», discipline dont il convient de signaler deux spécificités. L'une tient à son poids relativement faible dans le curriculum des centres d'apprentissage ${ }^{5}$, l'autre relève d'une tension structurante entre dépendance et émancipation par rapport aux disciplines techniques. Nous retenons, pour cette étude, la période qui va de 1945 à 1960, date à laquelle, sous l'effet de la mise en système de l'enseignement français opérée par la réforme Berthoin, les centres d'apprentissage deviennent des collèges d'enseignement technique (CET). Durant cette période, la

4 Ordonnance $\mathrm{n}^{\circ} 45-2630$ du 2 novembre 1945 portant création des Écoles normales nationales d'apprentissage. $J 0$ n ${ }^{\circ} 258$ du 2-3 novembre 1945, p.7192. L'ENNA de la rue de la Tour à Paris et celle de Toulouse sont plus spécifiquement considérées comme des ENNA féminines. Les ENNA se fondent dans les IUFM en 1991.

5 En 1945, sur les quarante-quatre heures par semaine passées trois en $2^{e}$ année et trois en $3^{e}$ année. méconnaissance par les acteurs de l'école de cette nouvelle filière d'enseignement et des établissements ayant pour mission de former ses enseignants a permis une autonomie favorable à l'expérimentation pédagogique et à la progressive construction d'un modèle disciplinaire se voulant différent de ceux de l'enseignement primaire et de l'enseignement secondaire.

Examiner dans quelle mesure les premières années d'existence des ENNA ont offert à leurs professeurs de français un contexte favorable pour penser la genèse et la structuration de cette discipline dans le cadre d'un projet éducatif visant à promouvoir un «humanisme du travail», tel est l'objet du présent article, qui s'inscrit dans une perspective historico-didactique (Bishop, 2013) permettant de saisir les enjeux de la scolarisation de la formation professionnelle et la déclinaison disciplinaire qui en est proposée. Pour ce faire, nous avons notamment exploité les archives des ENNA de Paris-Nord et de Nantes, conservées respectivement aux Archives nationales et aux archives départementales de Loire-Atlantique $^{6}$. Nous nous sommes également appuyée sur des textes officiels, des manuels scolaires, des sujets d'examens, ainsi que sur deux revues de première importance pour le monde de l'enseignement : la revue Technique, Art, Science, créée en 1946 par le ministère de l'Éducation nationale et la direction de I'Enseignement technique (DET), et Le Travailleur de l'enseignement technique, organe du Syndicat national de l'enseignement technique (SNET-CGT)'. Ces sources permettent en effet de décrire de manière fine le rôle des professeurs d'ENNA dans la mise en place de la discipline «français». Nous examinerons ainsi, dans une première partie, le projet culturel défendu par un petit groupe de professeurs d'ENNA proches de la direction de l'Enseignement technique et de son directeur, Paul Le Rolland ${ }^{8}$. Dans une

6 Les archives concernant I'ENNA de Paris-Nord (AN, 19771239/2) sont composées de plans de formation; celles relatives à l'ENNA de Nantes (AD 44, 1149 W 1-40) couvrent la période 1944-1979 et contiennent des pièces concernant l'ouverture et l'implantation de I'ENNA, des notes, des circulaires, des plans de formation, des épreuves d'examen de fin de stage, des comptes-rendus de visite et des appréciations de fin de stage. Ce travail s'inscrit dans un projet plus global d'exploitation des archives des cinq ENNA de Paris, Lyon, Nantes et Toulouse.

7 Le SNET, affilié à la CGT, crée en 1945 une section pour les centres d'apprentissage. Ce syndicat participe également à la commission Langevin-Wallon chargée de préparer la réforme de l'enseignement.

8 Paul Le Rolland fut membre de la commission Langevin-Wallon et directeur de l'Enseignement technique jusqu'en 1947. Membre du Parti communiste, il quitte cette fonction lors du départ des communistes du gouvernement. 
seconde partie, nous porterons plus spécifiquement notre attention sur les professeurs de la discipline « français » en nous intéressant, tout d'abord, à deux figures marquantes parmi les professeurs de français des ENNA et aux débats qui les opposèrent : Auguste Dumeix, qui enseigna à I'ENNA de Paris-Nord de 1945 à 1972, et André Rougerie, qui exerça à l'ENNA de Nantes de 1949 à 1963; en nous intéressant, ensuite, aux pratiques de formation en vigueur dans les ENNA, saisies à travers les examens d'entrée et de sortie, dont l'analyse permet de mieux comprendre la façon dont, à l'échelle locale, ces professeurs contribuent à structurer la discipline.

\section{Les professeurs d'ENNA et le projet éducatif de formation de l'homme, du citoyen et du travailleur}

En l'absence d'un recensement exhaustif des professeurs enseignant dans les ENNA, une estimation de la taille du groupe s'avère délicate. Les archives, actuellement dépouillées, présentent en effet des ruptures chronologiques qui empêchent de saisir les mouvements du personnel enseignant. Par ailleurs, les différences entre les ENNA en termes de sections, de nombre de stagiaires et d'organisation propre à chacune de ces écoles complexifient l'estimation que nous pouvons faire de ce groupe. À titre d'exemple, pour l'année 1946, I'ENNA de Paris-Nord compte 14 sections professionnelles alors que celle Nantes n'en accueille que 7. Toutefois, les éléments actuellement recueillis aux archives départementales de Loire-Atlantique, les plus précises pour la période qui nous intéresse, autorisent quelques projections quant au nombre d'intervenants. En 1950, 6 enseignants sont mentionnés pour les disciplines générales - 3 en français et 3 en mathématiques - et 25 pour les disciplines professionnelles. Concernant les professeurs de psychopédagogie, leur nom n'étant pas mentionné, il est difficile d'évaluer leur nombre. On peut faire I'hypothèse qu'une ENNA de la taille de celle de Nantes compte environ une trentaine de formateurs. On pourrait ainsi estimer qu'ils sont environ 150 à 200 à intervenir dans les ENNA avant 1960 et pour la seule discipline «français » proposer une estimation s'élevant à une vingtaine de personnes. L'analyse des discours de certains de ces professeurs ainsi qu'un retour sur les textes prescriptifs permettent de saisir leur rôle dans la consolidation du projet éducatif des ENNA.

\section{Les ENNA : un cadre nouveau mais des pratiques héritées}

Deux grandes catégories de maîtres destinés à enseigner dans les centres d'apprentissage sont formées dans les ENNA : d'une part, les professeurs d'enseignement technique théorique (PETT) et les professeurs techniques adjoints (PTA), et d'autre part les professeurs d'enseignement général (PEG), ces derniers étant des enseignants bivalents (PEG lettres-histoire et PEG lettres-langue vivante pour ce qui est de l'enseignement du français). Ces deux catégories sont recrutées dans des milieux professionnels différents : anciens ouvriers pour les enseignements techniques d'atelier, étudiants et surtout instituteurs pour les enseignements généraux. Leur formation à l'ENNA se déroule sous forme d'un stage d'une durée de 16 semaines jusqu'en 1950, puis d'une année ensuite. L'origine ouvrière des premiers et la connaissance qu'ont les seconds de l'éducation des enfants du peuple entrent en convergence avec le projet éducatif des ENNA, que les premiers professeurs de ces établissements portent sous le terme de culture ouvrière, nous y reviendrons.

Si les ENNA sont créées de toutes pièces, avec l'ambition de porter un modèle de formation professionnelle original, elles n'apparaissent toutefois pas vierges des modèles qui prévalaient dans la période antérieure à la Libération. Particulièrement prégnante est la «méthode Carrard», inspirée des travaux de l'ingénieur suisse et directeur de l'Institut psychotechnique de Lausanne Alfred Carrard, popularisée durant la Seconde Guerre mondiale dans les écoles formant les moniteurs ${ }^{9}$ des centres de formation professionnelle (CFP), notamment à l'école de la rue Dareau ouverte à Paris en 194010. Cette méthode, que Carrard suggérait même d'appliquer à l'enseignement du français (1942), repose sur la décomposition et la reproduction des gestes professionnels afin d'en favoriser l'acquisition rapide et de développer I'habileté manuelle. Imprégnés de cette méthode, les formateurs de la rue Dareau proposaient aux moniteurs des CFP des progressions standardisées et des exercices modélisants. Le but essentiel de la formation était de les convertir à l'esprit de la méthode. Comme l'écrit Bernard Bonnet (1999, p. 83) :

9 Le nom de moniteur était le nom donné aux formateurs des centres de formation professionnelle.

10 Une autre école, Belle-Ombre, ouvre près de ClermontFerrand. 
L'application des principes de la pédagogie Dareau aux adolescents a largement contribué au développement des CFP autant que ce dernier a favorisé leur diffusion massive auprès de milliers d'enseignants.

La survie de ce modèle parmi les enseignants des centres d'apprentissage et certains professeurs d'ENNA durant les années qui suivent la Seconde Guerre mondiale est attestée. Dans les Instructions sur les programmes et les méthodes des centres d'apprentissage de garçons parues en 1945, la référence à la méthode Carrard est explicite (p. 56). Un projet de 1945 d'organisation de la formation professionnelle pour l'industrie des cuirs et peaux, conservé aux archives départementales de Loire-Atlantique ${ }^{11}$, suggère aussi de recourir à cette méthode pour former les futurs enseignants d'une section «cuir» auxquels l'ENNA de Nantes ne peut proposer de formation.

\section{Une petite nébuleuse réformatrice à la manœuvre}

Dans ce contexte, certains professeurs d'ENNA, issus pour les uns de l'École normale supérieure de l'enseignement technique (ENSET) $)^{12}$ et pour les autres de I'École normale supérieure de Saint-Cloud, vont prendre position contre la méthode Carrard et proposer un modèle de formation original. C'est le cas de Fernand Canonge, professeur de psychopédagogie, Auguste Dumeix, professeur de français, et André Campa, professeur d'enseignement technique théorique, tous trois en poste à I'ENNA (masculine) de Paris. Proches de la direction de l'Enseignement technique et de son directeur Paul Le Rolland, ils font entendre leur voix dans les revues Technique, Art, Science et Le Travailleur de l'enseignement technique, mais aussi au sein de la commission Langevin-Wallon à laquelle participe Fernand Canonge, aux côtés de Paul Le Rolland ${ }^{13}$. Signalons enfin que tous sont membres ou proches du Parti communiste français, Auguste Dumeix étant chargé de mission auprès de Maurice Thorez en 1946. Ces professeurs, réunis dans la même institution, souhaitent défendre la noblesse de l'enseignement technique, peu considéré par les acteurs de l'enseignement

11 AD 44, 1149 W 1.

12 L'ENSET assure la formation des professeurs de l'enseignement technique se destinant à enseigner dans les Écoles nationales professionnelles (ENP) et les collèges techniques et non dans les centres d'apprentissage. Elle deviendra I'ENS de Cachan en 1985. secondaire, et faire des ENNA un maillon essentiel de la rationalisation de la formation des enseignants des centres d'apprentissage ainsi qu'un laboratoire de pratiques pédagogiques innovantes.

Ceci suppose, selon eux, de rompre avec l'héritage de Vichy et avec une méthode qu'ils jugent trop professionnelle et trop éloignée d'un projet de culture intégrale. La dénonciation explicite ou implicite de la méthode Carrard reparaît fréquemment sous leur plume, laissant penser, a contrario, que celle-ci perdurait chez des formateurs d'ENNA plus éloignés des principes portés par la direction de l'Enseignement technique ${ }^{14}$. En 1961, André Campa écrit ainsi dans la revue Technique, Art, Science que les ENNA ne forment pas de «simples répétiteurs, esclaves de leur formation » mais «préparent des professeurs chargés de l'enseignement des travaux d'ateliers et non des moniteurs» (Campa, 1961, p. 39). De même, Auguste Dumeix, son collègue de français, rappellera en 1997 , la rupture opérée par les ENNA pour mieux mettre en valeur la dimension culturelle de l'enseignement technique :

L'enseignement technique qui a été donné à partir de la création des ENNA constitue un tournant dans la formation. Il ne s'agit plus de savoir-faire on doit réfléchir pour savoir faire. Les savoir-faire, c'est l'apprentissage qui enseigne les gestes à faire avec les outils, avec l'aide d'un moniteur (p. 12).

Pour autant, le rejet de la méthode Carrard n'est pas synonyme d'alignement sur la culture scolaire du secondaire jugée trop livresque et reflétant, selon les instructions de 1945, une «conception bourgeoise » de la culture. Fernand Canonge rappelle ainsi, en 1947 :

Notre formule pédagogique ne nous viendra pas du dehors, pas plus du primaire que du secondaire, c'est nous qui la trouverons, qui élaborerons nos méthodes, nous qui sommes tous les jours au contact de nos jeunes gens (p. 2).

\section{Penser un humanisme du travail}

Cette formule pédagogique originale se place sous le signe d'un humanisme du travail qui inspire les textes officiels sur l'apprentissage et l'enseignement professionnel adoptés au lendemain de la Libération et à la conception desquels a participé Georges Friedmann,

14 La méthode Carrard a servi de référence pour la formation d'adultes. Hervé Terral (2005) estime, quant à lui, que cette méthode a continué d'exister en filigrane dans les discours pédagogiques de l'enseignement professionnel. 
sociologue et inspecteur général de l'Enseignement technique.

En effet, les conceptions de ce petit groupe de professeurs en matière de formation croisent les ambitions affichées dans les premiers textes officiels relatifs aux centres d'apprentissage. Les Instructions sur les programmes et les méthodes des centres d'apprentissage de garçons, parues en 1945, s'inscrivent dans la tradition de I'humanisme technique. Elles rappellent que l'enseignement, dans les centres d'apprentissage, «a un triple objet : humain, social et économique» et convoquent à cette fin la notion de culture ouvrière, déclinaison d'une culture technique pensée en fonction du destin social des élèves. Aussi la formation morale, la formation intellectuelle, la formation physique et la formation technique doivent-elles se fédérer autour du métier pour répondre aux enjeux d'une formation professionnelle scolarisée (Massat, 1958). II convient donc de ne pas envisager le futur travailleur du seul point de vue professionnel mais de chercher, dans une perspective d'émancipation, à faire fructifier en lui la valeur humaine que seule permet une éducation qui ne dissocie pas l'apprentissage du métier de la culture générale :

La nécessité d'une culture est évidente : il ne peut suffire d'apprendre au garçon ce qui est utile à l'exercice de son métier, mais bien de lui ouvrir l'accès aux satisfactions auxquelles il a droit en tant qu'ouvrier, Français et homme. La culture permet à chacun de dépasser la routine quotidienne à laquelle, s'il ne réagissait pas, il serait réduit, pensant et travaillant dans un monde irrémédiablement clos (Instructions sur les programmes et les méthodes des centres d'apprentissage de garçons, 1945, p. 12).

De la même façon, une circulaire du 7 mai 1945 explique :

L'apprentissage, tel que nous le concevons dans ces centres [d'apprentissage], est lui aussi, à sa manière, un enseignement complet et une éducation. Les centres, sans être des écoles, devront constituer à leur niveau des foyers d'«humanité technique», intégrés dans l'ensemble du système éducatif de la nation (BOEN n³4 du 7 juin 1945, p. 2202).

Cette vision est également celle qui anime le Comité de perfectionnement pédagogique de l'apprentissage, créé afin « d'étudier et de proposer toutes mesures susceptibles de contribuer au perfectionnement des méthodes pédagogiques en usage dans les centres d'apprentissage, aussi bien sur le plan de la formation technique que sur les plans des formations intellectuelles, morales et physiques $»^{15}$. Lors d'une réunion plénière du 7 décembre 1945, son président, Georges Friedmann (également proche du Parti communiste), invite précisément à réfléchir à la liaison entre les enseignements techniques et les enseignements généraux pour penser ce qu'il qualifie de culture ouvrière ${ }^{16}$ dans le cadre scolaire. Le projet de formation de l'homme, du citoyen et du travailleur ainsi énoncé repose donc sur une étroite relation entre les enseignements techniques et généraux. Ces enseignements contribuent à l'acquisition d'une culture scolaire qui ne peut relever des seules disciplines scolaires traditionnelles. Il convient, au contraire, «ne jamais se laisser entraîner par l'intérêt de chacune des disciplines", comme le rappellent les instructions de 1945 (p. 12). Ce projet est propre à valoriser le devenir social des élèves et à développer le désir de culture, comme l'écrit Fernand Canonge en 1947 :

Nous nous proposons [...] de servir la classe ouvrière et ses enfants, d'élever l'ouvrier, de lui donner le goût de la réflexion et de la connaissance qui permettent de dominer le métier. [...] Quand nos élèves nous quitteront il faut qu'ils aient encore l'appétit de connaître et la volonté de réaliser des progrès qui les rendront meilleurs dans tous les domaines (p. 2).

Le contenu des différents concours de recrutement de l'enseignement technique court et long confirme l'orientation spécifique donnée à la culture littéraire dans l'enseignement technique. Pour le recrutement des professeurs-formateurs de lettres, dont les modalités sont fixées par un arrêté du 25 février 1947, la deuxième épreuve écrite consiste en « une dissertation littéraire portant sur la littérature française du $18^{\mathrm{e}}$ $19^{\mathrm{e}}$ et $20^{\mathrm{e}}$ siècles envisagée du point de vue social ». Un sujet proposé en 1946 permet de comprendre la réflexion que l'on attend d'eux concernant la culture à donner aux futurs ouvriers :

Un critique contemporain s'est exprimé ainsi : «Si par la littérature vous entendez littérature écrite à l'intention du prolétariat, je prédis le plus déshonorant échec à ces livres qui prétendent aller au peuple. Si par littérature prolétarienne vous entendez : littérature de l'écrivain prolétarien, rival et successeur de l'écrivain bourgeois, je prends patience : le temps de cette littérature viendra. Mais si par littérature

15 Arrêté du 12 juillet 1945, JORF du 2 septembre 1945, p. 5507.

16 Il est à noter que, selon les lieux d'où ils parlent, les acteurs de l'enseignement technique court se réfèrent tantôt à I'humanisme du travail tantôt à la culture ouvrière sans jamais en souligner les points de divergence ou de convergence. 
prolétarienne vous entendez : littérature dont le prolétariat fournira le sujet, je vous dis : attention aux reportages». À la lumière de votre expérience et de vos lectures personnelles, approfondissez ce jugement et exposez votre point de vue.

Ce sujet révèle ainsi la volonté de faire des ENNA naissantes un lieu pour penser, dans le cadre scolaire, une culture qui serait plus populaire qu'ouvrière.

Les mêmes préoccupations transparaissent dans le cadrage initial du concours de recrutement des enseignants de français des centres d'apprentissage (PEG lettres-histoire et lettres-langues vivantes). Ce concours comprend à l'écrit une épreuve de composition de littérature sur une œuvre, un auteur ou une question figurant dans un programme révisé chaque année. Elle est suivie d'un commentaire grammatical et linguistique sur un passage d'un texte. Lors de l'épreuve orale, il est demandé aux candidats une explication de texte portant sur des œuvres également définies par un programme et une interrogation sur I'histoire littéraire française ${ }^{17}$. Les questions littéraires portent sur des sujets comme «La peinture de la condition ouvrière dans la littérature $d u X X^{e}$ siècle», "Les paysans dans la littérature française des $X X^{e}$ et $X X^{e}$ siècles» ou encore "Hugo, témoin de l'évolution sociale de son siècle». Elles permettent de prendre en compte la fonction sociale de la littérature et sa visée anthropologique et de concilier, comme le suggère Anne-Marie Thiesse (1991), la présence des auteurs du patrimoine, tout en travaillant l'image sublimée du peuple laborieux. La définition des épreuves de recrutement des PEG lettres est proche de celle du concours de recrutement à l'ENSET, qui forme les professeurs des collèges techniques et des écoles nationales professionnelles, et leurs programmes limitatifs sont parfois identiques. L'humanisme du travail apparaît donc, au départ, comme un horizon partagé de l'enseignement technique court et de l'enseignement technique long.

Ces orientations sont à resituer dans le contexte de 1945 et du projet d'éducation populaire tel qu'il est défendu par Peuple et Culture (1945) ou encore par l'un de ses soutiens, Jean Guéhenno, devenu inspecteur général de lettres en 1945. S'il s'agit de «rendre la culture au peuple et le peuple à la culture», il ne s'agit pas pour autant de réduire à la seule culture ouvrière une culture populaire, qui «ne saurait être qu'une culture commune à tout un peuple» (Manifeste de
17 Note du 18 janvier 1950, BOEN n 4 du 26 janvier 1950, p. 273-275.
Peuple et Culture). Dans la perspective de scolarisation à grande échelle de la formation ouvrière qui caractérise l'immédiat après-guerre, l'affirmation d'un modèle culturel ouvert sur la réalité socio-culturelle du temps apparaît ainsi comme le principe structurant d'un curriculum traversé par un projet de transmission d'une culture scolaire ouverte sur la vie et, pour partie, commun aux enseignements techniques court et long.

S'il met en exergue l'articulation entre les disciplines, ce projet éducatif et culturel global suppose aussi la stabilisation de pratiques, de contenus et de méthodes pour chacun des enseignements dispensés, et en particulier pour la discipline «français » étudiée dans cet article.

\section{Former à l'enseignement du français dans les ENNA : un modèle mal stabilisé ?}

Diverses sources permettent d'apprécier la contribution des professeurs d'ENNA à la structuration de la discipline «français » dans les centres d'apprentissage. L'analyse des publications - manuels ou articles des revues Technique, Art, Science et Le travailleur de l'enseignement technique - permet d'identifier les conceptions - en partie divergentes - défendues à titre individuel par deux d'entre eux, Auguste Dumeix et André Rougerie. L'analyse des plans d'études et des examens de sortie de l'ENNA - disponibles pour certains établissements - offre un aperçu direct des pratiques de formation. Le détail des concours de recrutement dans l'enseignement technique reflète, in fine, l'effet des transformations institutionnelles sur les (re)configurations disciplinaires.

\section{Deux figures marquantes de la discipline «français » et leurs divergences: Auguste Dumeix et André Rougerie}

Auguste Dumeix et André Rougerie, tous deux professeurs d'ENNA, se détachent plus particulièrement par le rôle qu'ils ont joué dans la configuration et la modélisation de la discipline "français», mais aussi pour leurs conceptions divergentes de la culture scolaire lorsqu'elle se donne pour finalité de former les futurs travailleurs.

Auguste Dumeix (1915-2002), professeur de français à I'ENNA de Paris, auteur régulier de la revue Technique, Art, Science, est également impliqué dans 
l'élaboration des programmes de français des centres d'apprentissage. Après avoir commenté les programmes de 1945 à la demande de l'inspection principale de l'académie de Montpellier (Dumeix, 1949), il participe à leur réécriture en 1954 ainsi qu'à la rédaction des instructions pédagogiques de 1960. En 1972 , il sera encore expert dans le groupe de travail chargé des programmes de $\mathrm{CAP}^{18}$. Sa proximité avec les corps d'inspection ainsi que sa participation dans la durée à la conception des programmes de l'enseignement professionnel sont un indicateur de son poids institutionnel.

La conception de la discipline «français» portée par Auguste Dumeix s'inscrit très clairement dans le projet humaniste de formation d'une jeunesse ouvrière porté par la direction de l'Enseignement technique. Les enjeux sociaux qui lui sont attachés sont pour lui centraux. Il s'agit de répondre aux besoins réels du futur travailleur et, comme le montre l'expression « mettre à disposition » dans la citation qui suit, l'enseignement du français est au service de l'apprenti et des pratiques qui seront les siennes dans son quotidien de travailleur et de citoyen :

De quoi s'agit-il ? De mettre à la disposition des futurs travailleurs la technique de l'expression orale et écrite et de meubler leur esprit des éléments de base qui leur permettront d'acquérir une culture [...] il va de soi que les lectures dites «professionnelles» étroitement liées à la technique devront être proscrites : les apprentis forgerons ne devront pas être condamnés pendant trois ans à une anthologie de la forge à travers la littérature française; le forgeron est forgeron mais il est aussi ouvrier, travailleur, citoyen, homme susceptible d'une vie privée et d'une vie publique qui dépasse de très loin, et fort heureusement, le labeur quotidien (Dumeix, 1949, p. 38-39).

L'article publié en 1946 par Dumeix dans Technique, Art, Science, sous le titre «Pour l'anthologie personnelle du professeur de français» (p. 19), permet d'appréhender la culture littéraire à proposer aux stagiaires des ENNA. On y retrouve, sous la forme de la lecture suivie, plusieurs textes d'auteurs également très présents dans les manuels des centres d'apprentissage : Le Notaire du Havre de Georges Duhamel, Train de vies d'Eugène Dabit et Le Passe-muraille de Marcel Aymé.

18 AN : 19920389/2. Au moment de sa mort en 2002, un article de L'Humanité du 9 janvier 2002 mentionne que Le Maitron, dictionnaire biographique du mouvement ouvrier et du mouvement social, précise que ce professeur a formé 630 professeurs d'enseignement général et 6000 professeurs techniques de CET-LEP.
Tous ces auteurs sont, pour lui, représentatifs de ce que pourrait être un programme d'auteurs permettant de répondre à un projet d'éducation populaire.

Professeur à l'ENP Livet de Nantes avant de devenir professeur à l'ENNA de cette ville en 1949, André Rougerie (1899-1994) est également auteur dans Technique, Art, Science, mais se remarque surtout par une activité éditoriale très dense ${ }^{19}$. Auteur de manuels depuis 1940, il publie des ouvrages de grammaire et une collection de "Textes choisis", dont chacun a fait l'objet de 4 à 5 rééditions jusqu'en 1992. Ces manuels s'adressent tantôt aux élèves des lycées et collèges ou des cours complémentaires, tantôt aux élèves des ENP, des collèges techniques et des centres d'apprentissage, tantôt à l'ensemble de ces filières réunies. L'analyse montre de grandes proximités entre ces divers ouvrages, tant dans le choix des textes que des démarches. Ce point mérite d'être souligné car, contrairement à son collègue Dumeix qui n'a pas écrit de manuels et qui s'est entièrement centré sur le français dans les centres d'apprentissage, André Rougerie pense la discipline dans la perspective d'une circulation et d'un rapprochement entre les différentes filières du second degré.

L'étude des manuels publiés par ce dernier entre 1950 et 1960 permet par ailleurs de cerner une évolution de la discipline «français» qui s'imposera au tournant des années 1960. Contrairement à son collègue Dumeix qui accorde aux écrivains ouvriers une place importante, André Rougerie recentre les corpus sur des textes plus proches de la culture scolaire du second degré général. Ceux-ci rompent nettement avec la veine ouvriériste et s'avèrent plus en adéquation avec les nouveaux programmes rédigés en 1954 dans la foulée de ceux des collèges techniques (1952) et dans lesquels la référence à la culture ouvrière a disparu. André Rougerie fait le choix de privilégier les récits d'aventures et de renforcer le poids des écrivains du $X V I^{e}$ siècle, plus particulièrement celui de Racine, faisant ainsi entrer la tragédie classique dans les corpus. Il affirme par ailleurs la présence de Maupassant, en tant que conteur, consacrant le poids de cet écrivain dans l'enseignement professionnel. Il tente ainsi de construire une image de la discipline sociologiquement

19 André Rougerie a également été membre expert dans une commission du Conseil de l'enseignement général et technique en juillet 1970, présidée par l'inspecteur général Josserand et consacrée à la révision des concours de recrutement des professeurs d'ENNA. On peut consulter le compte-rendu de ces travaux aux Archives nationales : AN 198110220/6 et 198110221/1. 
moins typée. Ses manuels annoncent ainsi une évolution de la discipline "français». Cette évolution est emblématique de l'affaiblissement de la littérature de tradition ouvrière dans l'enseignement technique et professionnel, très visible dans la période qui précède la Seconde Guerre mondiale mais incapable de se constituer en genre littéraire scolarisé. Dans la période qui va de 1945 à 1960, la progressive disparition des écrivains ouvriers dans les manuels révèle "une évolution silencieuse» de la discipline "français» (Lopez, 2015) ${ }^{20}$ amorcée dès la création des centres d'apprentissage. En tentant de construire une image sociologiquement moins typée, les manuels préparent «la perte d'identité de la discipline français» dans l'enseignement professionnel, que Lucie Tanguy, Catherine AguIhon et Françoise Ropé (1984) situent au tournant des années 1960, lorsque les centres d'apprentissage, devenus collèges d'enseignement technique, recruteront des élèves venant non plus des classes de fin d'études primaires mais du collège.

La revue Technique, Art, Science se fait, d'autre part, l'écho des divergences entre ces deux professeursformateurs au sujet de l'enseignement de la grammaire dont les modalités d'enseignement sont discutées dans les mêmes termes dans l'enseignement secondaire. Pour André Rougerie (1947), si le décloisonnement est nécessaire pour la lecture et l'écriture, la grammaire se doit d'être enseignée indépendamment des autres exercices de la classe de français. L'approche morphosyntaxique qu'il privilégie repose sur des séries d'exercices d'observation et de manipulation des faits de langue. Elle est très proche de celle utilisée dans la série des manuels qu'il publie chez Dunod à partir de 1950, Étude pratique de la langue française. Pour Auguste Dumeix, au contraire, l'étude de la grammaire ne peut se faire qu'à l'occasion de la lecture expliquée qui permet «naturellement »l'approfondissement d'un point de grammaire car, dit-il en réponse à André Rougerie, «la grammaire ne doit pas être enseignée comme un dogme, systématiquement» (Dumeix, 1947, p. 49) et doit reposer sur la collaboration entre les élèves et sur le développement de I'autonomie. Pour cela, Dumeix propose de leur fournir des fiches d'acquisition, de correction et de perfection-

20 Cette recherche porte sur 60 manuels de l'enseignement technique et professionnel entre 1930 et 1980 . Nous avons retenu plusieurs critères pour sélectionner les manuels : le nombre de rééditions, qui permet de faire I'hypothèse de leur visibilité, ainsi que le statut de leur concepteur, professeur d'ENNA, inspecteur, auteur nement. Cette démarche convient, selon lui, pour travailler la grammaire afin de développer les pratiques langagières des futurs ouvriers pour qu'ils puissent s'intégrer socialement.

La question des finalités et des modalités de l'étude de la langue semble d'ailleurs vive puisque dans cette même revue, une autre figure marquante de la discipline "français» dans les ENNA, Georges Court, professeur à l'ENNA de Paris-Nord et commentateur des sujets de CAP dans la revue le Cours industriel (19451983), participe au débat.

Notons qu'afin d'assurer la visibilité des pratiques de formation initiées dans les ENNA, la revue Technique, Art, Science s'ouvre aux productions des stagiaires qui reprennent les conseils méthodologiques donnés en formation. Un mois après l'article d'Auguste Dumeix, un stagiaire parisien rédige un article, "L'enseignement individualisé à l'aide de fiches» (Monjo, 1947, p. 33), qui se présente comme une exemplification des pratiques de formation initiées par ce formateur. Dans la même revue, deux autres proposent une programmation qui, à partir d'une approche par thèmes, organise la lecture, la grammaire et la composition française sous une forme globalisée mettant en œuvre le décloisonnement défendu par Dumeix dans La Revue pédagogique (1950). Dès les premières années d'existence des ENNA, les professeurs d'ENNA, ou du moins une partie d'entre eux, ont ainsi pu bénéficier du canal que constituait la revue à diffusion nationale Technique, Art, Science pour exposer et diffuser leur conception d'une didactique de la discipline «français» pour les centres d'apprentissage.

\section{Pratiques de formation en ENNA}

Présenter le rôle joué par les acteurs les plus visibles pour saisir la genèse de la discipline « français » ne suffit pas pour appréhender la modélisation qui en est proposée dans les ENNA. La consultation des archives des ENNA de Nantes et de Paris-Nord permet d'aller au-delà de la tribune que les revues ont pu offrir aux formateurs et de franchir le seuil de la «boîte noire». Dans quelle mesure les pratiques de formation dans ces ENNA répondent-elles, pour l'enseignement du français, au projet initial d'une "formule pédagogique originale» pour les centres d'apprentissage?

On a souligné l'importance accordée, dans les instructions officielles, à l'étroite relation devant exister entre enseignements techniques et généraux. L'étude des plans de formation de l'ENNA masculine de Paris à 
la fin des années $1950^{21}$ permet de voir concrètement comment cette recommandation est mise en œuvre dans la formation des professeurs stagiaires. Les professeurs de français sont formés à l'enseignement de la législation du travail pour ne pas ignorer le destin social de leurs futurs élèves et ne pas asseoir l'idée d'un cloisonnement disciplinaire qui ferait de la culture littéraire l'apanage des généralistes et, inversement, qui considérerait que toute culture professionnelle ne les concerne pas. Les professeurs stagiaires des spécialités techniques sont quant à eux sensibilisés à la culture littéraire et générale, comme le montre le contenu d'un cours de langue française proposé aux techniciens intitulé «Étude de questions d'orthographe, de grammaire et de vocabulaire sur un programme établi chaque année en fonction des besoins des stagiaires. Exercices pratiques d'élocution et de style». Un autre cours de culture littéraire consiste en la «présentation de quelques thèmes, auteurs ou œuvres, assortie de lecture commentée sur un programme établi chaque année en fonction des besoins des stagiaires». Enfin, pour le cours de culture générale, il est mentionné la «confection par les stagiaires de monographies simples sur des sujets d'enquête et de documentation fixés en accord avec eux».

Les contenus de formation accordent donc une place non négligeable à la construction d'une culture partagée et au nécessaire travail en équipe pédagogique pour garantir la finalité sociale d'une formation aux métiers et diffuser une culture scolaire ouverte sur le monde.

L'analyse des sujets d'examen proposés par les professeurs formateurs de français aux stagiaires lettres-histoire et lettres-langue de l'ENNA de Nantes ${ }^{22}$ entre 1948 et 1956 pour valider leur formation permet aussi de saisir la manière dont ces professeurs formateurs appréhendent la configuration de la discipline. Sur le plan pédagogique, son enseignement apparaît subordonné à une bonne connaissance des élèves, comme le montre ce sujet proposé en $1956^{23}$ :

Votre stage vous a permis de connaître les élèves des Centres d'Apprentissage et les conditions dans lesquelles ils travaillent. Dans quelle mesure y a-t-il une pédagogie propre aux Centres d'Apprentissage?

$1{ }^{\circ}$ En général

$2^{\circ}$ Pour l'enseignement de la composition française

21 AN 19771239/2. Recrutement et formation des maîtres (enseignement technique, écoles normales nationales d'apprentissage).

22 AD 44, 1149 W2, W3, W4 et W8.

23 AD 44, 1149 W 8.
Sur le plan didactique, les liens entre les sous-composantes de la discipline sont à privilégier, comme le stipule ce sujet donné en $1955^{24}$ :

Les programmes des centres d'apprentissage recommandent d'établir une liaison entre «explications de textes et devoirs de composition français ». Comment concevez-vous cette liaison? Vous vous appuierez sur des exemples précis.

Ce sujet fait écho à une proposition d'Auguste Dumeix qui, dans la Revue pédagogique (1950), présente «L'utilisation pédagogique des contes de Maupassant » sous la forme d'un tableau synoptique permettant de fédérer les activités de lecture, d'écriture et de langue autour d'un même objet. Cette organisation de la discipline «français» semble être un point de convergence entre les professeurs d'ENNA puisqu'on la retrouve également à l'ENNA féminine de Paris ${ }^{25}$. Cette mise en forme systématisée donne un aperçu de ce que pouvaient être les pratiques suggérées en formation pour les futurs enseignants des centres d'apprentissage.

Sur le plan culturel, enfin, les examens de fin de stage permettent de se projeter dans la classe en termes d'auteurs et d'activités proposées. Entre 1948 et 1956, les auteurs mentionnés sont Dautry, Guéhenno, Vercors et les thématiques des textes sont en relation avec l'éthique du travail qu'il convient de construire chez les élèves. L'une des questions posées à la fin du stage de $1948^{26}$ suite à la lecture d'un texte de Raoul Dautry, Métiers d'hommes, est ainsi formulée «Quel parti pédagogique tirerez-vous de ce texte du point de vue moral ?». Cette question est à rapprocher du principe de structuration des programmes autour de la formation morale, intellectuelle, physique et pratique. Ce lien est attesté par Lucien Dintzer, professeur de psychopédagogie à I'ENNA de Lyon :

Ainsi, mon collègue et ami, M. Paulin, professeur de lettres à I'ENNA de Lyon, a pu proposer récemment à nos stagiaires l'analyse du début de Crainquebille, le récit du pseudo-délit de l'infortuné marchand des quatre saisons. Les thèmes moraux abondent dans ce fragment. II convient d'en choisir un, deux au maximum, les adaptant au niveau et au goût des auditeurs (1948, p. 16).

L'analyse des sujets d'examen de fin de stage proposés par les professeurs d'ENNA permet ainsi de saisir les composantes de la discipline «français» que sont

24 AD 44, 1149 W 8.

25 AD 44, 1845 W 100.

26 AD 44, 1149 W 2. 
la lecture, l'étude du vocabulaire, la grammaire, qui ont pour finalité de vérifier la compréhension du texte par les élèves et la rédaction. En lecture, il est demandé de poser quelques questions sur le fond mais sans les multiplier. Le sujet de rédaction et les questions de vocabulaire et de grammaire doivent être inspirés par le texte. On retrouve ici une configuration très proche de celle des classes de fin d'études primaires d'où viennent la majorité des élèves des centres d'apprentissage. Dans la plupart des sujets, les textes étudiés constituent un réservoir pour les autres composantes. Le texte littéraire n'existe pas comme objet autonome à étudier pour lui-même et seulement un sujet sur 25 demande aux stagiaires d'imaginer une question sur l'intérêt littéraire du texte. Cette proximité entre le primaire et le technique court, qui peut apparaître assez éloignée du projet éducatif et de l'humanisme du travail défendus par les pionniers des ENNA, est attestée par d'autres sources : nous avons trouvé aux archives du Val-de-Marne les préparations d'un professeur de centre d'apprentissage à la fin des années $1950^{27}$. Celles-ci sont la recopie exacte du manuel Lectures actives publié en 1949 par un directeur d'école primaire, Maurice Duru. On peut donc faire l'hypothèse de la pérennité, chez certains PEG lettres, de pratiques proches de l'enseignement primaire.

Pris entre l'ambitieux projet éducatif des ENNA, les finalités de la formation dans les centres d'apprentissage et le niveau scolaire des élèves, les formateurs d'ENNA tentent d'inventer leur propre modèle au croisement de l'enseignement primaire, de l'enseignement secondaire et au regard des comparaisons avec I'ENSET. En l'absence de données permettant d'établir la proportion de PEG lettres passés par les ENNA, il reste toutefois difficile d'évaluer le degré de diffusion sur le terrain de la formation dispensée dans les ENNA.

\section{Une dynamique de secondarisation initiée par le technique long?}

Dans le courant des années 1950, divers signes attestent un glissement progressif, du projet initial singulier d'humanisme du travail vers un alignement de plus en plus marqué vers les normes et les pratiques de l'enseignement secondaire, suivant une trajectoire initiée par l'enseignement technique long.

27 Fond lycée professionnel Tony Garnier d'Alfortville, AD 94, 2153
En effet, dès les années 1950 s'amorce, pour les disciplines générales, un progressif alignement des professeurs de l'enseignement technique long (collèges techniques et ENP) sur le statut et les conditions de recrutement des professeurs de l'enseignement secondaire classique et moderne (Cardon-Quint, 2015). En 1954, les questions littéraires à consonance sociale - marqueurs de la spécificité d'une culture littéraire adaptée à l'enseignement technique - disparaissent du concours d'entrée à l'ENSET; le concours de recrutement des PEG lettres connaît la même évolution deux ans plus tard, en 1956. Signe que l'enseignement technique court est entraîné, par mimétisme, dans un mouvement de secondarisation initié par le technique long? Sans doute, mais cette évolution est aussi à mettre en relation avec celle du niveau de formation des candidats au professorat d'enseignement général des centres d'apprentissage. Majoritaires dans un premier temps, les instituteurs sont rapidement dépassés en nombre par des étudiants détenteurs d'un premier cycle universitaire. C'est par exemple le cas à l'ENNA de Nantes à la fin des années $1950^{28}$ où, sur vingt stagiaires, deux seulement sont des instituteurs, les autres ayant validé le certificat d'études littéraires à la fin du cycle de propédeutique ou même quelques certificats de licence. L'aménagement des concours de l'enseignement professionnel s'accorde donc également à l'évolution du vivier de candidats. L'élévation du niveau de formation des PEG lettres au cours des années 1950 ainsi que la spécialisation disciplinaire des épreuves du concours de recrutement éloignent les futurs stagiaires de l'enseignement bivalent auquel ils se destinent mais aussi du projet de formation intégrale reposant sur la solidarité entre les différentes disciplines que défendent les pionniers des ENNA.

Cette évolution du concours de recrutement des PEG lettres est à mettre en relation avec la définition de l'épreuve de lettres du concours de recrutement des formateurs d'ENNA. La mention «envisagée du point de vue social » a disparu au début des années 1960 de la définition de l'épreuve écrite du concours de recrutement des professeurs-formateurs de français. Elle devient «Dissertation littéraire portant sur la littérature française du XVI ${ }^{e}$ siècle à nos jours ». On peut ainsi faire I'hypothèse que la formation universitaire des futurs PEG lettres ainsi que les transformations des épreuves de concours de recrutement des futurs stagiaires des

28 AD 44, 1149 W 11 
ENNA et de leurs professeurs ont pu fragiliser le projet de transmission d'une culture populaire telle que I'avaient souhaitée les premiers professeurs de français des ENNA.

Enfin, dans les revues Techniques, Arts, Sciences et Le Travailleur de l'enseignement technique, les articles consacrés au projet éducatif et culturel de l'enseignement technique, à la pédagogie et aux disciplines générales se font plus rares dans les années 1950, pour disparaître à la fin de la décennie (Lamoure, 2010). La revue s'intéresse alors plus particulièrement aux enseignements pratiques essentiellement industriels, et les professeurs formateurs des disciplines littéraires s'effacent. Signe que l'alignement, pour les disciplines générales, sur le modèle offert par l'enseignement secondaire, est d'ores et déjà entériné?

\section{Conclusion}

Porter une attention particulière au rôle des acteurs a permis de saisir la manière dont un groupe de professeurs, proche de la direction de l'Enseignement technique, a tenté de construire et de diffuser un modèle disciplinaire dans le laboratoire que furent les ENNA.

Préservés par l'autonomie dont continuait à bénéficier l'enseignement technique, certains d'entre eux se sont montrés soucieux de concilier plusieurs influences. En pensant la discipline «français» dans la perspective d'un humanisme du travail adapté au devenir professionnel des élèves des centres d'apprentissage, ils ont tenté de préserver un modèle d'éducation intégrale fondé sur le décloisonnement disciplinaire.

La référence faite aux divergences de vues de deux figures emblématiques des ENNA a fait apparaître les évolutions silencieuses de la discipline «français » qui, en incorporant les contraintes inhérentes aux normes scolaires et disciplinaires, s'est peu à peu détachée du projet fondateur. Ces polémiques sont fondatrices des tensions qui traversent la discipline. Pour les uns, I'enjeu est de continuer à défendre le projet d'éducation populaire, pour les autres, il s'agit de se rapprocher d'un modèle plus en phase avec l'enseignement secondaire anticipant ainsi les évolutions institutionnelles à venir. La déprofessionnalisation de la discipline «français » et l'affaiblissement d'une approche systémique des disciplines qui s'ensuivirent peuvent en partie expliquer l'absence d'une réflexion sur une didactique de la bivalence dans les ENNA.

L'analyse du maillage d'acteurs pour appréhender la manière dont s'est ensuite diffusée et transformée la réflexion initiée par les pionniers des ENNA pourrait être poursuivie par l'étude de parcours individuels. Elle permettrait, par exemple, de travailler I'hypothèse d'une certaine porosité entre les professeurs d'ENNA et l'inspection générale de l'Enseignement technique. Pour des personnalités comme André Campa, professeur et sous-directeur de l'ENNA de Paris-Nord et inspecteur général de l'Enseignement technique de 1956 à 1974, ou comme René Basquin directeur de I'ENNA de Nantes de 1945 à 1949, devenu inspecteur général de l'Enseignement technique et ensuite directeur de I'ENSET jusqu'en 1970, les ENNA ont constitué une étape essentielle dans un parcours entièrement consacré à l'enseignement technique.

Le rôle structurant joué par les professeurs d'ENNA a continué au-delà de la période qui précède la réforme de 1960, puisque dans les années 1970-1980 ils sont encore très sollicités pour participer à la rédaction des programmes ou pour accompagner des expériences innovantes comme celle de la mise en place de la formation et de la certification par contrôle continu dans les années 1970. Mais, malgré cela, la visibilité de la réflexion didactique initiée dans les ENNA se réduit. La suppression en 1960 de la direction de l'Enseignement technique de l'organigramme du ministère de l'Éducation nationale et l'arrêt de la publication de la revue Technique, Art, Science en 1977 contribuent progressivement à la perte d'audience des professeurs d'ENNA et à l'effacement du projet culturel défendu dans les premières années d'existence de ces écoles.

Lorsqu'en 1991 les ENNA se fondent dans les Instituts universitaires de formation des maîtres (IUFM), certains de leurs acteurs, cités dans cet article, s'inquiètent. C'est le cas d'Auguste Dumeix qui s'exprime lors d'une journée organisée à Saint-Denis ${ }^{29}$ en 1991 à I'occasion des « 45 ans d'une instance de formation et de promotion des ouvriers en France : les ENNA» :

Relisant l'intitulé de la communication dont j'ai été chargée - je cite : «l'apport de l'expérience des ENNA aux IUFM [...]», je me demande si cet intitulé n'est pas prématuré. Pour qu'il y ait apport, il faudrait que les IUFM se le soient approprié. C'est très humblement ce que je leur souhaite. Car je souhaite que l'évocation de ce bientôt demi-siècle d'initiative pédagogique ne tourne pas à l'oraison funèbre, que vous le preniez comme un jalon et non comme une pierre tombale.

29 II s'agit de I'ENNA de Paris-Nord devenue alors un site IUFM chargé de la formation des professeurs des lycées professionnel et technologique. 
L'intégration de l'enseignement professionnel dans un système éducatif unifié et le rapprochement de la discipline "français» avec le modèle du second degré général ont favorisé le développement d'une didactique du français dans les ENNA. Toutefois, les travaux de référence dans ce domaine concernent essentiellement le premier degré et le second degré général. On peut alors se demander si la conséquence n'en fut pas la faible attention portée à la bivalence des professeurs de l'enseignement professionnel ${ }^{30}$ et

30 La réflexion sur la bivalence s'est développée récemment avec les travaux de Marie-France Rossignol (2017).

\section{Bibliographie}

ALLARD-GONDOUIN (2016). «La commission Langevin-Wallon et l'enseignement général dans la formation professionnelle initiale des employés, 1944-début des années 1950 ». In P. Kahn \& Y. Michel, Formation, transformations des savoirs scolaires. Caen : Presses universitaires de Caen, p. 247-257.

BISHOP M.-F. (2013). Statut et fonctions de la mise en perspective historique dans la didactique du français. Habilitation à diriger des recherches, sciences de l'éducation, université de Lille 3.

BONNET B (1999). La formation professionnelle des adultes: une institution et ses formateurs. Paris : L'Harmattan.

BRUCY G. (2005). «L'enseignement technique et professionnel français». Cahiers de la recherche sur l'éducation et les savoirs, $n^{\circ} 4$, p. 13-34. En ligne : <https://journals.openedition.org/cres/1226> (consulté le 20 septembre 2018).

CARDON-QUINT C. (2015). Des lettres au français. Une discipline à l'heure de la démocratisation (1945-1981). Rennes : Presses universitaires de Rennes.

LAMOURE J. (2010). «La revue technique, Art, Science, 19441955 : entre pédagogie et disciplines ». In R. d'Enfert \& P. Kahn, En attendant la réforme. Disciplines scolaires et politiques éducatives sous la IV République. Grenoble : Presses universitaires de Grenoble, p. 160-168.

LÉON A. (1983). Histoire de l'éducation populaire. Paris : Nathan.

LOPEZ M. (2014). «Les écoles normales nationales d'apprentissage et la formation des maîtres dans l'enseignement professionnel » In A. Prost, La formation des maîtres de 1940 à 2010. Rennes: Presses universitaires de Rennes, p. 105-118.

LOPEZ M. (2015). Formation littéraire et formation professionnelle de 1930 à 1985. Thèse de doctorat, sciences du langage, université de Cergy-Pontoise.

PELPEL P. \& TROGER V. (1993). Histoire de l'enseignement technique. Paris : Hachette-Éducation. l'abandon d'une réflexion sur le projet éducatif et sur la culture scolaire à proposer aux élèves, très majoritairement issus des milieux populaires et se destinant à des études professionnelles.

Maryse Lopez

Université de Cergy-Pontoise et ESPE de Versailles, laboratoire ÉMA (EA 4507) maryse.lopez@u-cergy.fr

Dictionnaire des concepts fondamentaux de la didactique. Bruxelles : De Boeck, p. 85-88.

ROSSIGNOL M.-F. (2017). «Intervalence et didactique du français dans la formation en master "lettres-histoire-géographie" et "lettres-langues"». Le français aujourd'hui, n¹99, p. 67-77.

SOREL E. (1997). Une ambition pour l'école. Le plan Langevin-Wallon. Paris : Éditions sociales.

TANGUY L. (1991). L'enseignement professionnel en France. Des ouvriers aux techniciens. Paris : PUF.

TANGUY L., AGULHON C. \& ROPÉ F. (1984). «L'enseignement du français en LEP, miroir d'une perte d'identité». Études de linguistique appliquée, $\mathrm{n}^{\circ}$ 54, p.19-38.

TERRAL H. (2002). "Les Écoles Normales Nationales d'Apprentissage (1945-1991), Une institution-clé dans la formation des maîtres". Recherche et formation, $n^{\circ} 40$, p. 141-157.

TERRAL H. (2005). «Alfred Carrard (1889-1948) et la formation du travailleur». In T. Charmasson (dir.), Formation au travail, enseignement technique et apprentissage. Actes du 127 Congrès national des sociétés historiques et scientifiques, organisé à Nancy, du 15 au 20 avril 2002. Paris : CTHS, p. 87-95.

THIESSE A. M. (1991). Écrire la France, le mouvement littéraire régionaliste de langue française entre la belle époque et la Libération. Paris : PUF.

TROGER V. (1991). Histoire des centres d'apprentissage, 19391959 : les enjeux économiques, politiques et culturels de la constitution de l'enseignement technique court. Thèse de doctorat, histoire, université Paris IV-Sorbonne.

TROGER V. (1998). «La formation des enseignants dans l'enseignement professionnel depuis 1945 : les contradictions d'une pratique pionnière ». In R. Bourdoncle \& L. Demailly, Les professions de l'éducation et de la formation. Villeneuve-d'Ascq : Presses universitaires du Septentrion, p. 91-99.

TROGER V. (1999). «De l'éducation populaire à la formation 
professionnelle, l'action de Peuple et Culture». Sociétés contemporaines, n³5, p.19-42.

\section{Sources}

CAMPA A. (1961). «La formation des professeurs techniques adjoints ». Technique, Art, Science, $\mathrm{n}^{\circ} 145$, janvier, p.38-39.

CARRARD A. (1942). La jeunesse de demain : réforme scolaire, principes pédagogiques, Neuchâtel; Paris : Delachaux et Niestlé.

CANONGE F. (1946). «Les centres font partie du second degré». Technique, Art, Science, $\mathrm{n}^{\circ}{ }^{1}$, octobre, p.7-9.

CANONGE F. (1947). «Les centres dans la réforme Langevin ». Le Travailleur de l'enseignement technique, $\mathrm{n}^{\circ} 14$, mai, p. 1-2.

DINTZER L. (1948). «L'enseignement de la législation». Technique, Art, Science, $\mathrm{n}^{\circ}$ 4, janvier 1948, p. 36.

DUMEIX A. (1946). «Pour une anthologie personnelle du professeur de français », Technique, Art, Science, n ${ }^{1}$, octobre, p.18-19.

DUMEIX A. (1947). «Les fiches pédagogiques dans l'enseignement de la langue française ». Technique, Art, Science, $\mathrm{n}^{\circ} 4$, janvier, p. 49-51.

DUMEIX A (1949). «L'esprit et les formes de l'enseignement du français dans les Centres d'apprentissage de garçons». Technique, Art, Science, nº 1, octobre, p. 38-42.

DUMEIX A. (1950). «L'utilisation pédagogique des contes de Maupassant». Revue pédagogique, $\mathrm{n}^{\circ} 1$, octobre, p. 9-11.

DUMEIX A. (1997). "Formation des maîtres. Hier-aujourd'hui-demain». Les Cahiers de I'IRETEP, n³6, janvier-mars, p. 12.

DURU M. (1949). Lectures actives. Livre unique pour l'enseignement du français dans les classes et dans les centres d'apprentissage. Paris : Hachette.

GAL R. (1946). «Le dernier congrès de l'Éducation nouvelle ». Technique, Art, Science, nº2, novembre, p. 9.

LÉON A. (1965). Formation générale et apprentissage du métier, essai sur la psychopédagogie de l'enseignement technique. Paris : PUF

MIALARET G. (1997). Plan Langevin Wallon. Paris : PUF.
MASSAT A (1958). «La coordination des disciplines dans les établissements de l'enseignement technique». Technique, Art, Science, $\mathrm{n}^{\circ} 114$, janvier, p. 53-58.

MONJO L. (1947). «L'enseignement individualisé à l'aide de fiches ». Technique, Art, Science, $\mathrm{n}^{\circ}$ 4, février, p. 33.

NAVILLE (1948). «La formation professionnelle et l'école». Paris : PUF.

PEUPLE ET CULTURE (1945). Un peuple, une culture, manifeste de Peuple et Culture. En ligne : <http://www.peuple-etculture.org/IMG/pdf/manifeste_peuple_et_culture.pdf> (consulté le 20 septembre 2018).

ROUGERIE A. (1947). «L'enseignement de la grammaire». Technique, Art, Science, $\mathrm{n}^{\circ} 4$, janvier, p. 44-45.

\section{Sources administratives}

DIRECTION DE L'ENSEIGNEMENT TECHNIQUE (1945). Instructions sur les programmes et les méthodes des centres d'apprentissage de garçons. Lyon : France-empire.

MINISTÈRE DE L'ÉDUCATION NATIONALE (1954). Centres d'apprentissage. Enseignement industriel. Garçon. Enseignements généraux. Paris : IPN

Arrêté du 18 septembre 1944 relatif à l'organisation de l'enseignement technique. JO n०88 du 4 octobre 1944, p. 864-876.

Arrêté du 12 juillet 1945 relatif à la création d'un comité de perfectionnement pédagogique de l'apprentissage. BOEN n ${ }^{\circ} 42$ du 23 août 1945, p.2959-2960.

Circulaire du 7 mai 1945 relative à l'organisation des centres d'apprentissage. BOEN n³4, 7 juin 1945, p. 2202-2204.

Loi du 21 février 1949 relative au statut des centres d'apprentissage. JO n 46 du 22 février 1949, p. 1943-1944.

Note du 18 janvier 1950 relative à la mise en place d'un concours de recrutement pour les professeurs de CET. BOEN n ${ }^{\circ} 4 \mathrm{du}$ 26 janvier 1950, p. 273-275.

Ordonnance $n^{\circ}$ 45-2630 du 2 novembre 1945 portant création des Écoles normales nationales d'apprentissage. JO n² 258 du 2-3 novembre 1945, p.7192. 
